Cellular Physiology
and Biochemistry and Biochemistry Published online: December 09, 2015

Accepted: November 09, 2015

This article is licensed under the Creative Commons Attribution-NonCommercial-NoDerivatives 4.0 International License (CC BY-NC-ND) (http://www.karger.com/Services/OpenAccessLicense). Usage and distribution for commercial purposes as well as any distribution of modified material requires written permission.

Original Paper

\title{
Crocetin Activates Foxp3 Through TIPE2 in Asthma-Associated Treg Cells
}

\author{
Jurong Ding ${ }^{\mathrm{a}}$ Jianhua Su ${ }^{\mathrm{b}} \quad$ Li Zhang $^{\mathrm{a}} \quad$ Jian Ma ${ }^{\mathrm{a}}$ \\ aDepartment of Emergency, Shanghai Pulmonary Hospital, Tongji University of Medicine, Shanghai, \\ bDepartment of Respiration, Huashan Hospital Baoshan Branch, Fudan University School of Medicine, \\ Shanghai, China
}

\section{Key Words}

OVA - Asthma - Crocetin - Regulatory T cells (Treg) - Foxp3 - Tumor necrosis factor (TNF)alpha-induced protein 8-like 2 (TIPE2)

\begin{abstract}
Background/Aims: Regulatory T cells (Treg) are critical regulators of asthma. Crocetin is isolated from Chinese herb saffron and is a natural carotenoid dicarboxylic acid with antiinflammatory potential. However, the effects of Crocetin on asthma as well as the underlying mechanisms have not been studied. Methods: We used Crocetin to treat mice with established ovalbumin (OVA)-induced asthma. We purified CD4+CD25+ Treg cells by flow cytometry and analyzed the levels of two immunoregulatory proteins Foxp3 and tumor necrosis factor (TNF)-alpha-induced protein 8-like 2 (TIPE2) in Treg cells. We depleted either Foxp3 or TIPE2 in mouse lung through lentivirus-mediated delivery of shRNA, and analyzed their effects on severity of asthma and Treg cells after Crocetin treatment. Results: Crocetin treatment significantly reduced the severity of an ovalbumin (OVA)-induced asthma in mice. Moreover, Crocetin significantly increased the levels of TIPE2 and Foxp3 in Treg cells and the number of Treg cells. Depletion of Foxp3 abolished the increased in Treg cells, and the effects of Crocetin on the severity of asthma, without affecting TIPE2 levels in Treg cells. On the other hand, depletion of TIPE2 abolished both the increased in Treg cells and the effects of Crocetin on the severity of asthma, through suppressing Foxp3. Conclusion: Crocetin may activate Foxp3 through TIPE2 in asthma-associated Treg cells to mitigate the severity of asthma.

\section{Introduction}

Typical pathological events in Asthma include bronchial inflammation, airway hyperresponsiveness and airflow obstruction. Allergic asthma makes up most asthma cases,

J. Ding and J. Su contributed equally to this work.

Li Zhang and Jian Ma

KARGER 125
Department of Emergency, Shanghai Pulmonary Hospital, Tongji University of Medicine, 507 Zhengmin Road, Shanghai 200433, (China)

Tel.+862165115006, E-Mail pangzilama@gmail.com and E-Mail majian_15@163.com 


\section{Cellular Physiology Cell Physiol Biochem 2015;37:2425-2433 \begin{tabular}{l|l|l|l|l} 
DOI: 10.1159/000438595 & (c) 2015 The Author(s). Published by S. Karger AG, Basel \\
www.karger.com/cpb
\end{tabular} \\ Ding et al.: Crocetin Regulates Treg Cells Through TIPE2-Mediated Foxp3 in Asthma}

characterized by activation of type-2 T helper cells, IgE production, eosinophilia, and an inappropriate T cell response to environmental allergens [1-4].

Regulatory T cells (Treg) are T cells that suppress potentially harmful immune responses, and have been shown to suppress inflammatory responses and airway hyper-responsiveness to maintain peripheral immune tolerance [5-7]. Treg has been defined by surface markers of combined CD4 and CD25 and also characterized by expression of a member of the forkhead box transcription factor, Foxp3 [7-9]. Foxp3 is crucial for naive T cell differentiation towards the Treg phenotype and its expansion [7-14]. However, the molecular bases underlying the activation of Treg through Foxp3 are not completely understood.

Previous studies have shown that herb-drug could improve the therapeutic effects and reduce side effects during cancer treatment. Saffron is a Chinese traditional herb, and Crocetin is the major constituent of saffron, which has recently found to have multiple pharmacological effects including anticancer [15-17]. However, the effects of Crocetin on asthma as well as the underlying mechanisms have not been studied.

Tumor necrosis factor (TNF)-alpha-induced protein 8-like 2 (TIPE2) was first identified as a novel immune negative molecule that regulates the adaptive and innate immunity $[18,19]$. TIPE2 was later found to inhibit inducible nitric oxide synthase (iNOS) and thus generation of nitric oxide, resulting in suppression of inflammation [20-27]. The dysregulation of TIPE2 has also been found to mediate diverse immunological diseases [28-32]. However, whether TIPE2 may be involved in the pathogenesis of asthma and regulation of Treg has not been reported.

In the current study, we studied the effects of Crocetin treatment on the severity of an ovalbumin (OVA)-induced asthma in mice, and in this model, we studied the relationship between TIPE2 and Foxp3 in Treg cells.

\section{Materials and Methods}

\section{Mouse handling}

All mouse experiments were approved by the IACUC of Shanghai Pulmonary Hospital. Only 10-weekold male C57BL/6 mice (Jackson lab, Bar Harbor, ME, USA) were used for in vivo experiments. Mice were housed in a specific pathogen-free environment.

Ovalbumin (OVA)-induced allergic asthma model and Crocetin treatment

Male C57BL/6 mice of 10 weeks of age were sensitized with an intraperitoneal injection of $50 \mu \mathrm{g}$ OVA (OVA, grade V; Sigma-Aldrich, St. Louis, MO, USA) with $2 \mathrm{mg}$ aluminum hydroxide gel (Alum; SigmaAldrich) once per week for 3 times (week 0-2). Then the mice were challenged with $50 \mu \mathrm{g}$ OVA by intranasal administration under light anesthesia every other day for another 7 weeks (week 3-9). Control mice received PBS. After another week, the mice were examined, sampled and analyzed (week 10). Crocetin $\left(\mathrm{C}_{20} \mathrm{H}_{24} \mathrm{O}_{4}\right.$, molecular weight, 328.4, MP Biomedicals, Santa Ana, CA, USA) in $100 \mu \mathrm{l}$ dimethyl sulfoxide (DMSO) was given by intranasal administration after OVA challenge daily at a dose of $100 \mu \mathrm{mol} / \mathrm{l}$ till the end of the experiment (week 9-10).

\section{Airway hyper-responsiveness}

Airway hyper-responsiveness (AHR) was measured by restrained invasive plethysmography 1 day after the last intranasal OVA challenge. Mice were anesthetized, after which a small incision was made to expose the trachea, and a cannula was inserted to connect to an inline nebulizer and ventilator. Mice were then challenged with aerosolized PBS followed by increasing doses of methacholine (Sigma-Aldrich). Airway resistance and dynamic compliance (Cdyn) were determined by analysis of pressure and flow waveforms.

Bronchoalveolar lavage, lung digestion and isolation of Treg

Mice were euthanized by pentobarbitone overdose after AHR examination. Bronchoalveolar lavage fluid (BALF) was obtained by instilling three washes of $0.4 \mathrm{ml} \mathrm{PBS}$ with $0.1 \%$ BSA. BALF was centrifuged at $470 \mathrm{~g}$ for $5 \mathrm{~min}$, and cells were enumerated and labeled for further analysis. For lung digestion, lungs were 


\section{Cellular Physiology Cell Physiol Biochem 2015;37:2425-2433 \begin{tabular}{l|l|l} 
and Biochemistry & Dublished online: December 09, 2015 & $\begin{array}{l}\text { (c) } 2015 \text { The Author(s). Published by S. Karger AG, Basel } \\
\text { www.karger.com/cpb }\end{array}$
\end{tabular} \\ Ding et al:: Crocetin Regulates Treg Cells Through TIPE2-Mediated Foxp3 in Asthma}

perfused with PBS, after which $0.8 \mathrm{ml} 300 \mathrm{U} / \mathrm{ml}$ collagenase type I (Sigma-Aldrich), and $50 \mathrm{U} / \mathrm{ml} \mathrm{DNase}$ I (Roche, Nutley, NJ, USA) in RPMI 1640 was injected into the trachea. Lungs were then removed, minced into small pieces, and digested at $37^{\circ} \mathrm{C}$ for $30 \mathrm{~min}$. Lung pieces were disrupted with a syringe plunger, filtered through nylon mesh, and centrifuged. The cell pellet was resuspended in RBC lysis buffer, washed, enumerated, and labeled for flow cytometric analysis. Treg was analyzed and purified by fluorescenceactivated cell sorting (FACS) with specific fluorescence-conjugated antibodies (CD4 and CD25, all from Becton-Dickinson Biosciences, San Jose, CA, USA) on a FACSAria cell sorter (Becton-Dickinson Biosciences). Data were analyzed using Flowjo software (Flowjo LLC, Ashland, OR, USA).

\section{Lentivirus production and in vitro and in vivo transduction}

We used a pcDNA3.1-CAG-GFP plasmid as backbones (all from Clontech, Mountain View, CA, USA). The sequence for shTIPE2 is 5'-CCG TGT AAA CCA CTC AAC TCA TCTT-3', and the sequence for shFoxp3 is 5'-GCA CAT TCC CAG AGT TCCT-3'. The plasmids and target shRNA (or control scrambled sequence-scr) were digested with Xhol and BamHI and subcloned with a 2A into a pcDNA3.1-CAG-luciferase, resulting in a construct named pCAG-shTIPE2-2A-GFP or pCAG-shFoxp3-2A-GFP. The small 2A peptide sequences, when cloned between genes, allow for efficient, stoichiometric production of discrete protein products within a single vector through a novel "cleavage" event within the 2A peptide sequence. Sequencing was performed to confirm the correct orientation of the new plasmid. To generate lentiviral particles, HEK293T cells were seeded in a $100 \mathrm{~mm}$ dish at 50,000 cells $/ \mathrm{cm}^{2}$ and co-transfected with $10 \mu \mathrm{g}$ of target plasmids and $5 \mu \mathrm{g}$ each of packaging plasmids (REV, pMDL and VSV-G) using Lipofectamine-2000 (Invitrogen). The supernatant containing lentiviral particles was collected 48 hours after transfection and filtered through a $0.45 \mu \mathrm{m}$ syringe filter. The viruses were purified using $\mathrm{CsCl}$ density centrifugation and then titered by a quantitative densitometric dot-blot assay. For cell transduction in vitro, Treg were seeded in $100 \mathrm{~mm}$ plates at 15,000 cells $/ \mathrm{cm}^{2}$ one day prior to lentiviral infection. The lentiviral particles were added along with $10 \mu \mathrm{g} / \mathrm{ml}$ polybrene (Sigma-Aldrich) to the cell culture at a multiplicity of infection (MOI) of 100 for 48 hours. Then the cells were washed twice with complete media and the transduction efficiency ( $>90 \%$ ) was evaluated by GFP. For in vivo transduction, the $10^{6}$ virus was injected via tail vein.

\section{RT-qPCR}

Total RNA was extracted from purified Treg by FACS or mouse lung, using RNeasy (Qiagen, Hilden, Germany), and cDNA synthesis was performed by reserve transcription. Quantitative PCR (RT-qPCR) was performed in duplicates with QuantiTect SYBR Green PCR Kit (Qiagen). All primers were purchased from Qiagen. Data were collected and analyzed using $2^{-\Delta \Delta C t}$ method for quantification of the relative mRNA expression levels. Values of genes were first normalized against $\beta$-actin, and then compared to experimental controls.

\section{Statistics}

Statistical analysis was performed with the unpaired two-tailed Student t test (for comparison between two groups), one-way ANOVA with the Tukey posttest (for comparison between three or more groups), or repeated-measures ANOVA with the Dunnett posttest (for AHR dose-response curves), using GraphPad Prism software (GraphPad Software, Inc. La Jolla, CA, USA). Data were represented as mean \pm SD and were considered significant if $\mathrm{p}<0.05$.

\section{Results}

\section{Crocetin attenuates OVA-induced hallmarks of the asthma}

We used OVA-induced mouse asthma model in our study. In this model, mice were first sensitized to alum-adsorbed OVA for 2 weeks, and then exposed to repeated airway provocation for 7 weeks to develop AHR. Crocetin was given after OVA challenge for one week, and then the mice were analyzed (Fig. 1A). The establishment of asthma model was demonstrated by a dose-dependent increase in lung resistance ( $\mathrm{Rl}$ ) and decrease in Cdyn in response to a cholinergic stimulus (methacholine), which were significantly attenuated by Crocetin treatment (Fig. 1B-C). Another hallmark feature of OVA-induced allergic asthma 
Fig. 1. Crocetin attenuates OVA-induced hallmarks of the asthma. (A) Experimental schematic for OVA sensitization, inhalation treatment. (B-C) RI (B) and Cdyn (C) in response to increasing doses of methacholine. (D) Percentage of leukocytes in BALF. ${ }^{*} \mathrm{p}<0.05$, $\mathrm{N}=10$. Statistics: oneway ANOVA with a Bonferroni Correction. CRO: Crocetin. CTL: Control. challenge, and Crocetin

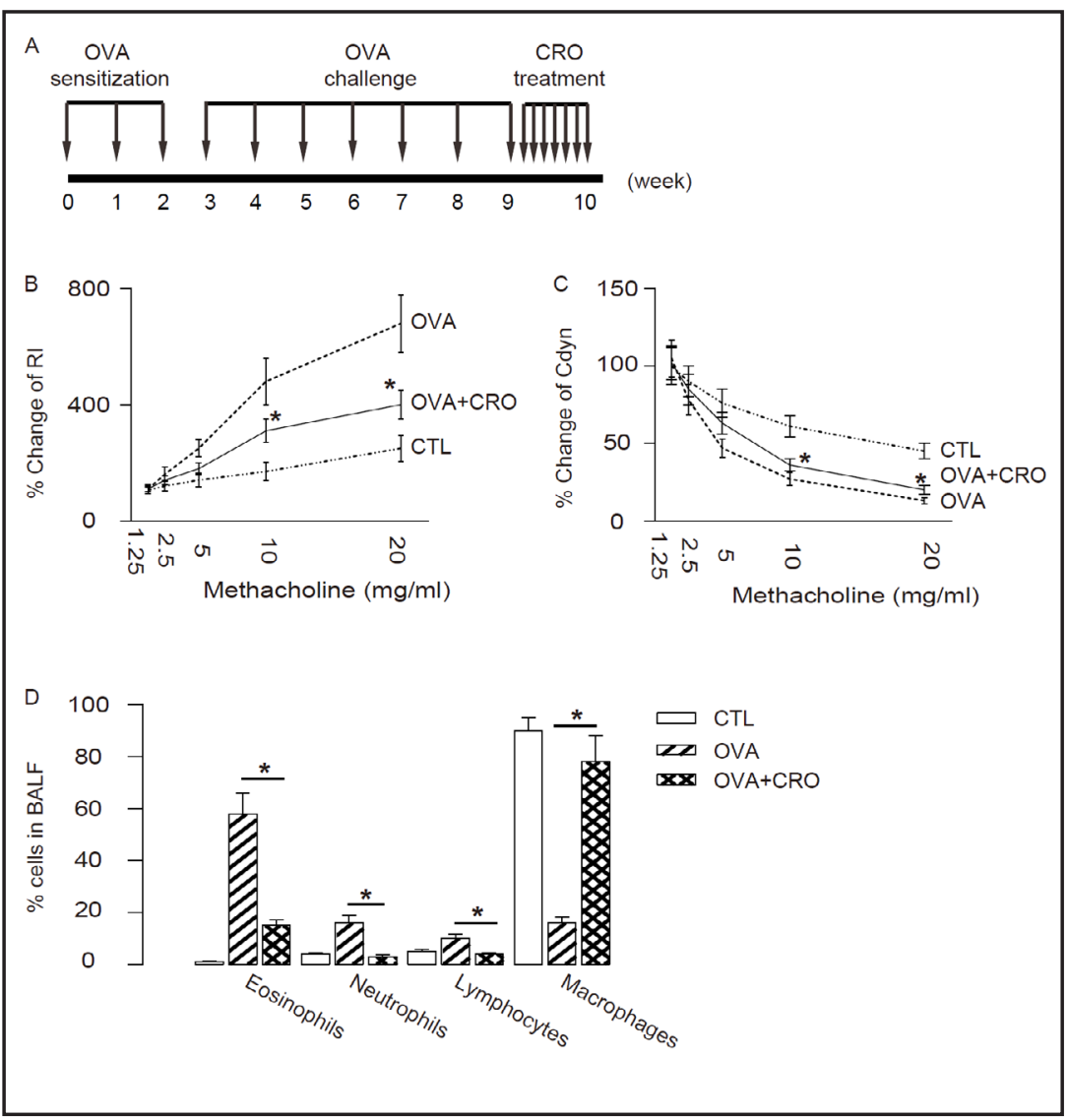

Fig. 2. Crocetin increases Treg cells in OVA-treated mouse lung. (A-B) Treg cells were purified from the lung digests of OVA/Crocetin-treated mice based on CD4 and CD25 positivity by flow cytometry, shown by representative flow charts (A), and by quantification (B). (C) Foxp3 and TIPE2 levels in Treg cells by RT-qPCR. *p < 0.05. NS: non-significant, $N=10$. Statistics: one-way ANOVA with a Bonferroni Correction. CRO: Crocetin. CTL: Control.

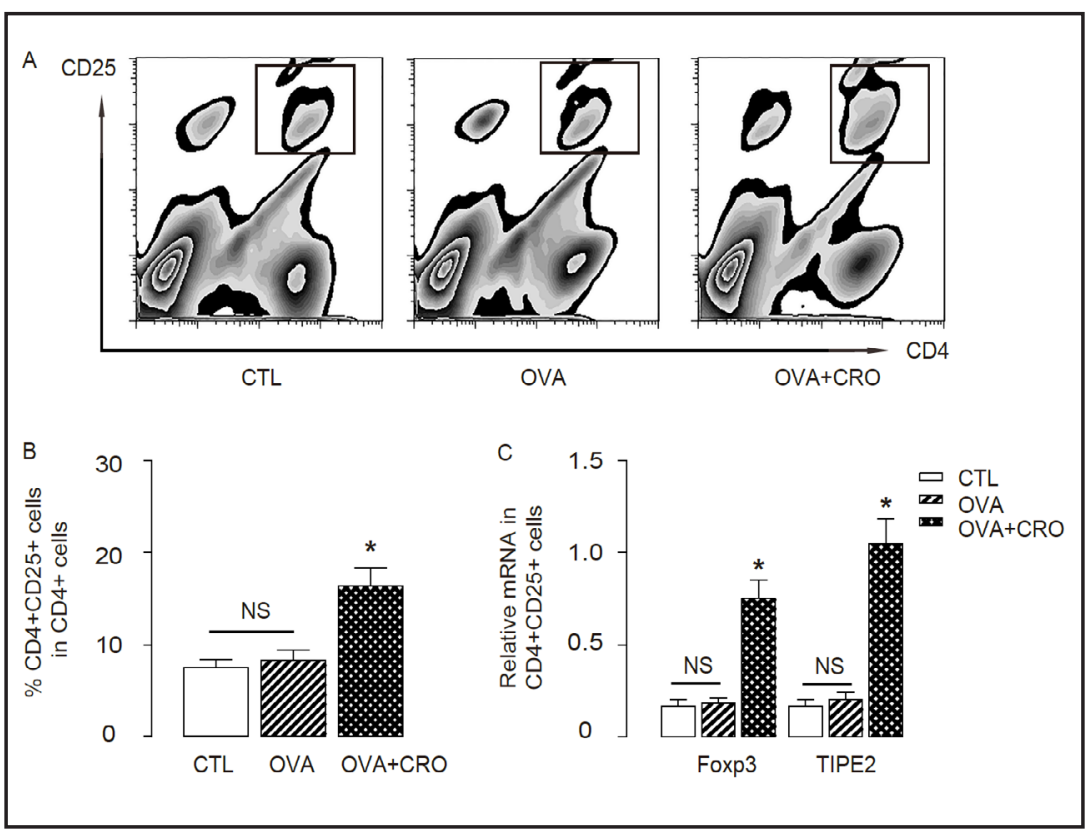

-eosinophilic accumulation in the pulmonary airways- was also evaluated. We found that the influx of inflammatory eosinophils increased to about $60 \%$ of total BALF cells by OVAtreatment, which was also significantly attenuated by Crocetin treatment (Fig. 1D). These data suggest that Crocetin attenuates OVA-induced hallmarks of the asthma in mice.

\section{KARGER}


Fig. 3. Depletion of Foxp3 abolishes the effects of Crocetin on OVA-asthma without affecting TIPE2. We generated a lentivirus carrying shFoxp3 or a scrambled sequence control (scr). (A-B) These viruses were first used to transduce primary mouse CD4+CD25+ Treg in vitro. RT-qPCR for Foxp3 (A) and TIPE2 (B) were performed. (C) Experimental schematic for OVA sensitization, inhalation challenge, Crocetin treatment, and viral treatment. (D-E) Treg cells were purified from the lung digests based on CD4 and CD25 positivity by flow cytometry,

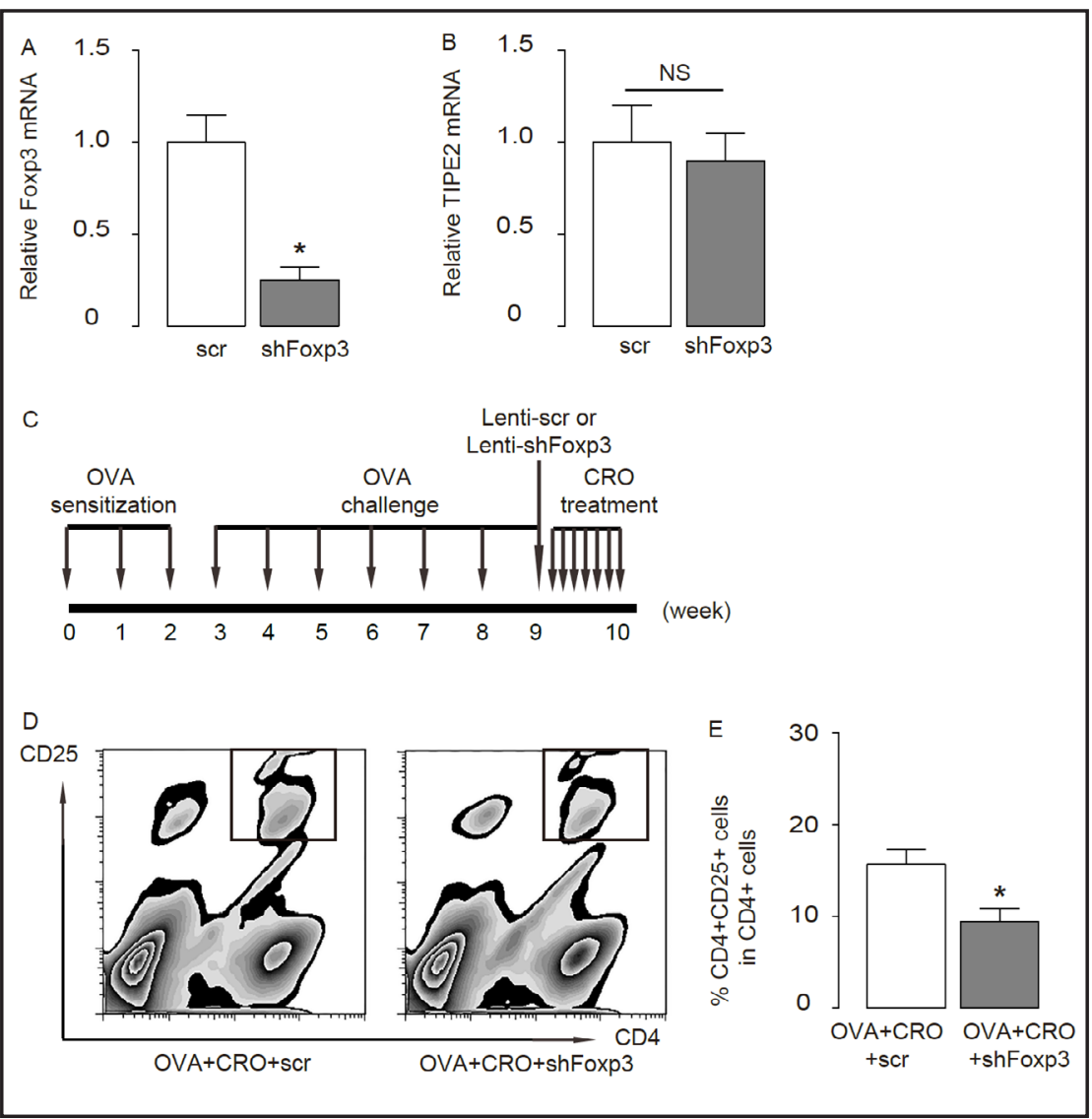
shown by representative flow charts (D), and by quantification (E). (F-G) RI (F) and Cdyn (G) in response to increasing doses of methacholine. ${ }^{*} \mathrm{p}<0.05$. NS: non-significant, $\mathrm{N}=10$. Statistics: one-way ANOVA with a Bonferroni Correction. CRO: Crocetin. CTL: Control.

\section{Crocetin increases Treg cells in OVA-treated mouse lung}

Then we aimed to find out the molecular mechanisms underlying the therapeutic effects of Crocetin. We hypothesized that Treg cells may be regulated by Crocetin, since Treg cells are potent inflammation suppressor in asthma. We used CD4 and CD25 to purify Treg from the lung digests of the mice by flow cytometry (Fig. 2A). We found that OVA itself did not significantly increase Treg, but we detected a significant increase in Treg cell number in the Crocetin-treated mouse lung (Fig. 2B). Interestingly, we found that the levels of both Foxp3 and TIPE2 significantly increased in CD4+CD25+ Treg cells from OVA+Crocetin treated mouse lung, compared to control or OVA-only-treated mice (Fig. 2C). Thus, Crocetin increases Treg in OVA-treated mouse lung, and the levels of Foxp3 and TIPE2 in Treg.

\section{Depletion of Foxp3 abolishes the effects of Crocetin on OVA-asthma without affecting TIPE2}

In order to examine the role of Foxp3 and TIPE2 in Crocetin-treated OVA-asthma, we generated a lentivirus carrying shFoxp3 or a scrambled sequence control (scr). These viruses were first used to transduce primary mouse CD4+CD25+ Treg in vitro. First, the knock-down of Foxp3 in Treg by shFoxp3 was confirmed by RT-qPCR (Fig. 3A). We found that depletion of Foxp3 in Treg did not alter TIPE2 levels (Fig. 3B). Then, these viruses were given to mice at 9 week, immediately before Crocetin treatment (Fig. 3C). We found that depletion of Foxp3 by lentivirus abolished the increases in Treg by Crocetin (Fig. 3D-E), resulting in abolishment of the effects of Crocetin on RI (Fig. 3F), and Cdyn (Fig. 3G). These data suggest that depletion of Foxp3 abolishes the effects of Crocetin on OVA-asthma and Treg, without affecting TIPE2.

\section{KARGER}


Fig. 4. Depletion of TIPE2 abolishes the effects of Crocetin on OVA-asthma via Foxp3. We generated a lentivirus carrying shTIPE2 or a scrambled sequence control (scr). (AB) These viruses were first used to transduce primary mouse C D 4 + C D 25 + Treg in vitro. RT-qPCR for TIPE2 (A) and Foxp3 (B) were performed. (C) Experimental schematic for OVA sensitization, inhalation challenge, Crocetin treatment, and viral treatment. (D-E) Treg cells were purified from the lung digests based on CD4 and CD25 positivity by flow cytometry, shown by representative
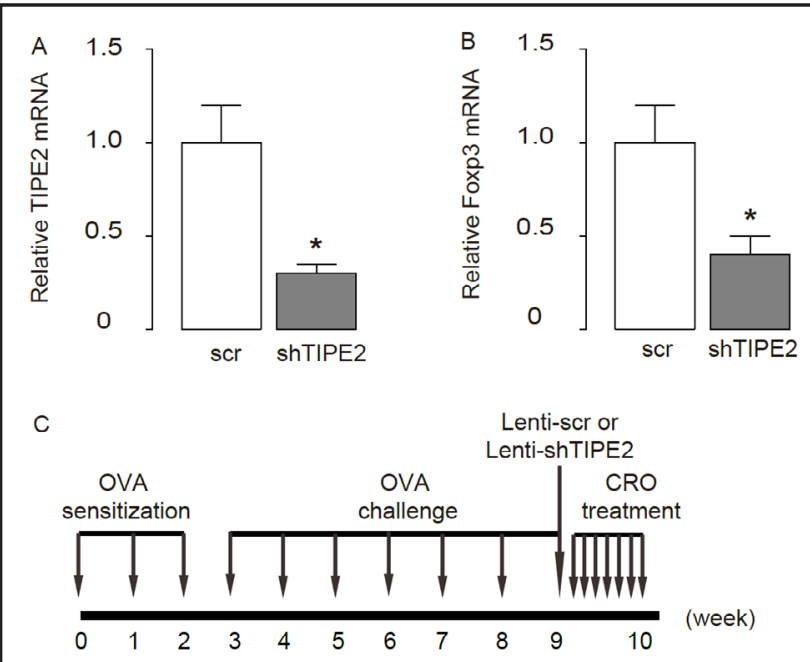

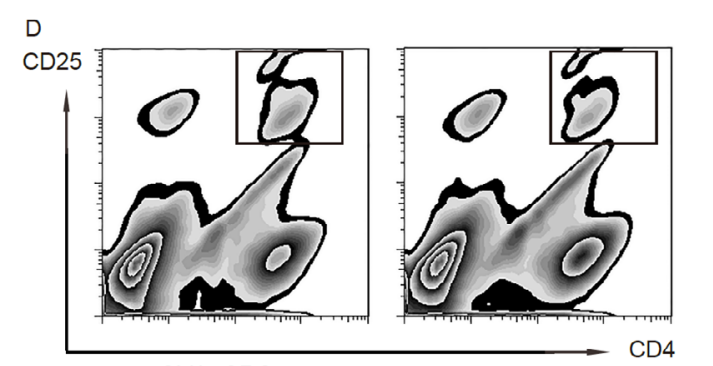

OVA+CRO+scr

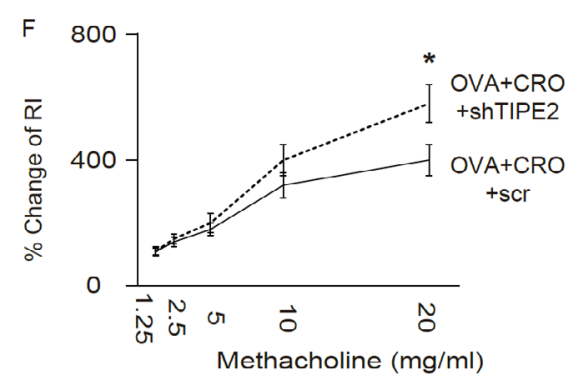

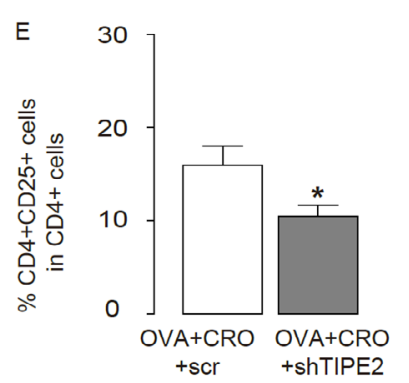

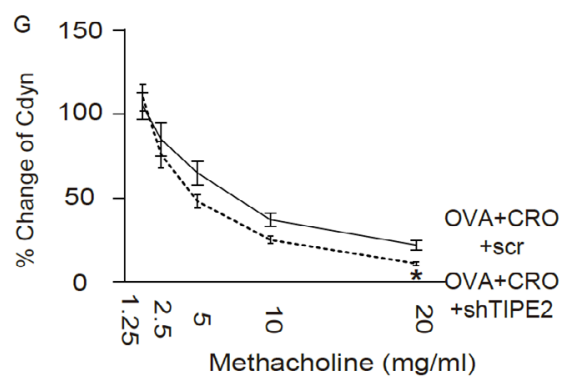

flow charts (D), and by quantification (E). (F-G) RI (F) and Cdyn (G) in response to increasing doses of methacholine. ${ }^{*} \mathrm{p}<0.05, \mathrm{~N}=10$. Statistics: one-way ANOVA with a Bonferroni Correction. CRO: Crocetin. CTL: Control.

\section{Depletion of TIPE2 abolishes the effects of Crocetin on OVA-asthma via Foxp3}

Next, we generated a lentivirus carrying shTIPE2 or a scrambled sequence control (scr). These viruses were first used to transduce primary mouse CD4+CD25+ Treg in vitro. First, the knock-down of TIPE2 in Treg by shTIPE2 was confirmed by RT-qPCR (Fig. 4A). We found that depletion of TIPE2 in Treg significantly decreased Foxp3 levels (Fig. 4B). Then, these viruses were given to mice at 9 week, immediately before Crocetin treatment (Fig. 4C). We found that depletion of TIPE2 by lentivirus abolished the increases in Treg by Crocetin (Fig. 4D-E), resulting in abolishment of the effects of Crocetin on RI (Fig. 4F), and Cdyn (Fig. 4G). These data suggest that depletion of TIPE2 abolishes the effects of Crocetin on OVA-asthma and Treg, possibly through Foxp3. Together, our data suggest that Crocetin may increase Foxp3 through TIPE2 to activate Treg to suppress the severity of asthma (Fig. 5). 


\section{Cellular Physiology and Biochemistry Published online: December 09,2015 $\begin{aligned} & \text { (c) 2015 The Author(s). Published by S. Karger AG, Basel } \\ & \text { www.karger.com/cpb }\end{aligned}$ \\ Ding et al.: Crocetin Regulates Treg Cells Through TIPE2-Mediated Foxp3 in Asthma}

Fig. 5. A schematic model. Crocetin may increase Foxp3 through TIPE2 to activate Treg to suppress the severity of asthma.

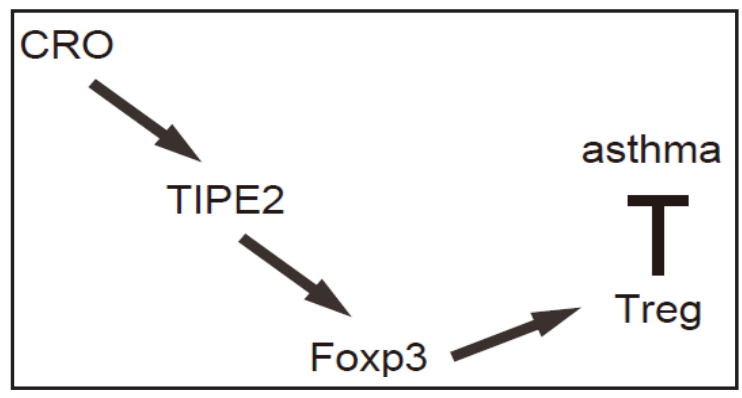

\section{Discussion}

Crocetin is a sort of carotenoids that have been reported of a lot of biomedical properties including anticancer [33, 34]. Nevertheless, its regulation on inflammatory responses has not been extensively studied. Especially, a role of Crocetin in asthma treatment is not acknowledged. Here, we sought to determine the role of Crocetin in asthma control, as well as the critical cellular and molecular signaling that mediate the activation of Treg cells by Crocetin to alleviate allergic asthma.

TIPE2 is a recently detected molecular with anti-inflammatory potential. TIPE2 is an essential negative regulator of inflammation and immune homeostasis. TIPE2-deficent mice suffer from chronic inflammatory diseases and TIPE2-deficient T cells and macrophages produce significantly increased levels of inflammatory cytokines. The inflammatory disease in TIPE2-deficent mice shares many similarities with those that have Foxp3 deficiency [35]. Thus, a regulatory relationship between TIPE2 and Foxp3, although never examined, deserves exploration.

We used a well-established mouse allergic asthma model [23], and purified CD4+CD25+ Treg. First, we showed that Crocetin attenuated OVA-induced hallmarks of the asthma. Next, we found that the number of Treg was increased by Crocetin. Since Foxp3 is a regulator of Treg activation and amplification, we examined the levels of Foxp3 in Treg, and found that Foxp3 was significantly increased by Crocetin. Moreover, we also examined the levels of TIPE2 in Treg, and found that it similarly upregulated after Crocetin treatment. Hence, we thought that both TIPE2 and Foxp3 may be involved in the effects of Crocetin on Treg. To prove it, we knocked out either TIPE2 or Foxp3 in Treg, and found that the Foxp3 levels were regulated by TIPE2, but not vice versa. Thus, it seemed that TIPE2 was upstream of Foxp3 and this pathway was activated upon Crocetin treatment. Furthermore, the block of this pathway at either TIPE2 or Foxp3 level completely abolished the activation of Treg by Crocetin.

Together, our data suggest that Crocetin may increase Foxp3 through TIPE2 to activate Treg to suppress the severity of asthma, and reveal a previously unrecognized crosstalk between TIPE2 and Foxp3 in the regulation of Treg to affect the severity of asthma, specifically after Crocetin treatment. Thus, our studies may shed light on TIPE2 as a novel therapeutic target for treating allergic asthma.

\section{Disclosure Statement}

The authors have declared that no competing interests exist.

\section{References}

1 Holgate ST: Pathogenesis of asthma. Clin Exp Allergy 2008;38:872-897.

2 Holgate ST: The airway epithelium is central to the pathogenesis of asthma. Allergol Int 2008;57:1-10. 


\section{Cellular Physiology Cell Physiol Biochem 2015;37:2425-2433 \begin{tabular}{l|l|l}
\hline DOI: 10.1159/000438595 & (c) 2015 The Author(s). Published by S. Karger AG, Basel \\
wwwwkarger.com/cpb
\end{tabular} \\ Ding et al.: Crocetin Regulates Treg Cells Through TIPE2-Mediated Foxp3 in Asthma}

3 Meng K, Zhang W, Zhong Y, Mao X, Lin Y, Huang Y, Lang M, Peng Y, Zhu Z, Liu Y, Zhao X, Yu K, Wu B, Ji Q, Zeng Q: Impairment of circulating cd4(+)cd25(+)garp(+) regulatory t cells in patients with acute coronary syndrome. Cell Physiol Biochem 2014;33:621-632.

$4 \quad$ Kraczyk B, Remus R, Hardt C: Cd49d treg cells with high suppressive capacity are remarkably less efficient on activated cd45ra- than on naive cd45ra+ teff cells. Cell Physiol Biochem 2014;34:346-355.

5 Schreiber TH, Wolf D, Tsai MS, Chirinos J, Deyev VV, Gonzalez L, Malek TR, Levy RB, Podack ER: Therapeutic treg expansion in mice by tnfrsf25 prevents allergic lung inflammation. J Clin Invest 2010;120:3629-3640.

6 Doganci A, Eigenbrod T, Krug N, De Sanctis GT, Hausding M, Erpenbeck VJ, Haddad el B, Lehr HA, Schmitt E, Bopp T, Kallen KJ, Herz U, Schmitt S, Luft C, Hecht O, Hohlfeld JM, Ito H, Nishimoto N, Yoshizaki K, Kishimoto T, Rose-John S, Renz H, Neurath MF, Galle PR, Finotto S: The il-6r alpha chain controls lung cd4+cd25+ treg development and function during allergic airway inflammation in vivo. J Clin Invest 2005;115:313-325.

7 Trzonkowski P, Szmit E, Mysliwska J, Mysliwski A: Cd4+cd25+t regulatory cells inhibit cytotoxic activity of ctl and nk cells in humans-impact of immunosenescence. Clin Immunol 2006;119:307-316.

8 Pyzik M, Piccirillo CA: Tgf-beta1 modulates foxp3 expression and regulatory activity in distinct cd4+t cell subsets. J Leukoc Biol 2007;82:335-346.

9 Palomares O, Ruckert B, Jartti T, Kucuksezer UC, Puhakka T, Gomez E, Fahrner HB, Speiser A, Jung A, Kwok WW, Kalogjera L, Akdis M, Akdis CA: Induction and maintenance of allergen-specific foxp3+ treg cells in human tonsils as potential first-line organs of oral tolerance. J Allergy Clin Immunol 2012;129:510-520, 520 e511-519.

10 Soroosh P, Doherty TA, Duan W, Mehta AK, Choi H, Adams YF, Mikulski Z, Khorram N, Rosenthal P, Broide $\mathrm{DH}$, Croft M: Lung-resident tissue macrophages generate foxp3+ regulatory t cells and promote airway tolerance. J Exp Med 2013;210:775-788.

11 Aggarwal NR, Tsushima K, Eto Y, Tripathi A, Mandke P, Mock JR, Garibaldi BT, Singer BD, Sidhaye VK, Horton MR, King LS, D'Alessio FR: Immunological priming requires regulatory t cells and il-10-producing macrophages to accelerate resolution from severe lung inflammation. J Immunol 2014;192:4453-4464.

12 Coleman MM, Ruane D, Moran B, Dunne PJ, Keane J, Mills KH: Alveolar macrophages contribute to respiratory tolerance by inducing foxp3 expression in naive t cells. Am J Respir Cell Mol Biol 2013;48:773780.

13 Zaslona Z, Przybranowski S, Wilke C, van Rooijen N, Teitz-Tennenbaum S, Osterholzer JJ, Wilkinson JE, Moore BB, Peters-Golden M: Resident alveolar macrophages suppress, whereas recruited monocytes promote, allergic lung inflammation in murine models of asthma. J Immunol 2014;193:4245-4253.

14 Tran DQ, Ramsey H, Shevach EM: Induction of foxp3 expression in naive human cd4+foxp3 t cells by t-cell receptor stimulation is transforming growth factor-beta dependent but does not confer a regulatory phenotype. Blood 2007;110:2983-2990.

15 Li S, Jiang S, Jiang W, Zhou Y, Shen XY, Luo T, Kong LP, Wang HQ: Anticancer effects of crocetin in human esophageal squamous cell carcinoma kyse-150 cells. Oncol Lett 2015;9:1254-1260.

16 Kim SH, Lee JM, Kim SC, Park CB, Lee PC: Proposed cytotoxic mechanisms of the saffron carotenoids crocin and crocetin on cancer cell lines. Biochem Cell Biol 2014;92:105-111.

17 Gadgoli C, Shelke S: Crocetin from the tubular calyx of nyctanthes arbor-tristis. Nat Prod Res 2010;24:1610-1615.

18 Zhang X, Wang J, Fan C, Li H, Sun H, Gong S, Chen YH, Shi Y: Crystal structure of tipe2 provides insights into immune homeostasis. Nat Struct Mol Biol 2009;16:89-90.

19 Sun H, Gong S, Carmody RJ, Hilliard A, Li L, Sun J, Kong L, Xu L, Hilliard B, Hu S, Shen H, Yang X, Chen YH: Tipe2, a negative regulator of innate and adaptive immunity that maintains immune homeostasis. Cell 2008;133:415-426.

20 Lou Y, Zhang G, Geng M, Zhang W, Cui J, Liu S: Tipe2 negatively regulates inflammation by switching arginine metabolism from nitric oxide synthase to arginase. PLoS One 2014;9:e96508.

21 Xiao X, Gaffar I, Guo P, Wiersch J, Fischbach S, Peirish L, Song Z, El-Gohary Y, Prasadan K, Shiota C, Gittes GK: M2 macrophages promote beta-cell proliferation by up-regulation of smad7. Proc Natl Acad Sci USA 2014;111:E1211-1220.

22 Xiao Q Zhang X, Wu Y, Yang Y: Inhibition of macrophage polarization prohibits growth of human osteosarcoma. Tumour Biol 2014;35:7611-7616. 


\section{Cellular Physiology Cell Physiol Biochem 2015;37:2425-2433 \begin{tabular}{ll|l} 
DOI: 10.1159/000438595 & $\begin{array}{l}\text { O } 2015 \text { The Author(s). Published by S. Karger AG, Basel } \\
\text { www.karger.com/cpb }\end{array}$
\end{tabular} \\ Ding et al.: Crocetin Regulates Treg Cells Through TIPE2-Mediated Foxp3 in Asthma}

23 Song X, Xie S, Lu K, Wang C: Mesenchymal stem cells alleviate experimental asthma by inducing polarization of alveolar macrophages. Inflammation 2015;38:485-492.

24 Wang B, Miao Y, Zhao Z, Zhong Y: Inflammatory macrophages promotes development of diabetic encephalopathy. Cell Physiol Biochem 2015;36:1142-1150.

25 Pan B, Liu G, Jiang Z, Zheng D: Regulation of renal fibrosis by macrophage polarization. Cell Physiol Biochem 2015;35:1062-1069.

26 Liu W, Zhang S, Gu S, Sang L, Dai C: Mesenchymal stem cells recruit macrophages to alleviate experimental colitis through tgfbeta1. Cell Physiol Biochem 2015;35:858-865.

27 Lin S, Qiu M, Chen J: Il-4 modulates macrophage polarization in ankylosing spondylitis. Cell Physiol Biochem 2015;35:2213-2222.

28 Li D, Song L, Fan Y, Li X, Li Y, Chen J, Zhu F, Guo C, Shi Y, Zhang L: Down-regulation of tipe2 mrna expression in peripheral blood mononuclear cells from patients with systemic lupus erythematosus. Clin Immunol 2009;133:422-427.

29 Xi W, Hu Y, Liu Y, Zhang J, Wang L, Lou Y, Qu Z, Cui J, Zhang G, Liang X, Ma C, Gao C, Chen Y, Liu S: Roles of tipe2 in hepatitis b virus-induced hepatic inflammation in humans and mice. Mol Immunol 2011;48:12031208.

30 Zhang Y, Wei X, Liu L, Liu S, Wang Z, Zhang B, Fan B, Yang F, Huang S, Jiang F, Chen YH, Yi F: Tipe2, a novel regulator of immunity, protects against experimental stroke. J Biol Chem 2012;287:32546-32555.

31 Kong L, Liu K, Zhang YZ, Jin M, Wu BR, Wang WZ, Li W, Nan YM, Chen YH: Downregulation of tipe2 mrna expression in peripheral blood mononuclear cells from patients with chronic hepatitis c. Hepatol Int 2013;7:844-849.

32 Ma Y, Liu X, Wei Z, Wang X, Wang Z, Zhong W, Li Y, Zhu F, Guo C, Zhang L, Wang X: The expression and significance of tipe2 in peripheral blood mononuclear cells from asthmatic children. Scand J Immunol 2013;78:523-528.

33 Gutheil WG, Reed G, Ray A, Anant S, Dhar A: Crocetin: An agent derived from saffron for prevention and therapy for cancer. Curr Pharm Biotechnol 2012;13:173-179.

34 Bathaie SZ, Farajzade A, Hoshyar R: A review of the chemistry and uses of crocins and crocetin, the carotenoid natural dyes in saffron, with particular emphasis on applications as colorants including their use as biological stains. Biotech Histochem 2014;89:401-411.

35 Luan YY, Yao YM, Zhang L, Dong N, Zhang QH, Yu Y, Sheng ZY: Expression of tumor necrosis factor-alpha induced protein 8 like-2 contributes to the immunosuppressive property of $\operatorname{cd} 4(+) \operatorname{cd} 25(+)$ regulatory $t$ cells in mice. Mol Immunol 2011;49:219-226. 